\title{
Clinical implications of GWAS variants associated with differentiated thyroid cancer
}

\author{
Jarosław Jendrzejewski ${ }^{1}$,Krzysztof Sworczak', Daniel F. Comiskey Jr2, Albert de la Chapelle ${ }^{2}$ \\ ${ }^{1}$ Department of Endocrinology and Internal Medicine, Medical University of Gdansk, Gdansk, Poland \\ ${ }^{2}$ Human Cancer Genetics Program, Comprehensive Cancer Centre, The Ohio State University, Columbus, Ohio, United States
}

\begin{abstract}
The genetic risk of differentiated thyroid cancer (DTC) probably consists of multiple low-penetrance, single-nucleotide polymorphisms (SNP). Such markers are difficult to uncover by linkage analysis but can be revealed by association studies. Genome-wide association studies (GWASs) have uncovered 31 SNPs associated with DTC. These markers carry a low to moderate risk for DTC, but their cumulative effect increases with each successive risk allele. These data support the important contribution of low penetrance variants in the pathogenesis of DTC. Contrary to somatic mutations such as BRAFV600E, germline variants can be ascertained prior to surgical treatment. Therefore, we hypothesise that GWAS SNPs might impact the clinical course of DTC and we can benefit from this knowledge in choosing a treatment strategy. Several associations between clinical factors and GWAS markers have been reported so far. The most important are associations between $\mathrm{rs} 966423$ and mortality $(\mathrm{HR}=1.60, \mathrm{p}=0.038)$, extrathyroidal extension (ETE) $(\mathrm{OR}=1.57, \mathrm{p}=0.019)$; rs965513 and tumour diameter (slope of regression $0.14, \mathrm{p}=0.025)$, lymph node metastasis $(\mathrm{OR}=1.59, \mathrm{p}=0.030)$ and ETE $(\mathrm{OR}=1.29, \mathrm{p}=0.045)$; rs944289 and distant metastasis $(\mathrm{OR}=0.58, \mathrm{p}=0.042)$; and rs116909374 and lymph node metastasis $(\mathrm{OR}=0.61, \mathrm{p}=0.016)$. These findings show that GWAS SNPs are not only the ignition factors (together with environmental factors) for malignant transformation of thyrocytes but might also impact the clinical course of DTC. Surprisingly, it is not always the risk allele for DTC that is associated with worse clinical outcome. The second interesting observation is that GWAS SNPs show different associations with DTC clinical features depending on their histological subtypes. These point to the complexity of DTC with putatively different roles of genes at different stages of DTC development. (Endokrynol Pol 2019; 70 (5): 423-429)
\end{abstract}

Key words: genome-wide association studies; GWAS; papillary thyroid carcinoma; PTC; genetic predisposition

\section{Introduction}

Thyroid carcinoma (TC) displays one of the strongest familiarities among cancers. Large case-control studies analysing populations from Utah and Sweden showed that the family risk ratio (FRR) for first-degree relatives of probands was 8.48 and 12.42 , respectively [1-3]. Such data suggest a strong genetic component in TC development. However, it remained unclear whether the reported familiarity in TC was due to the dominant role of genes or the influence of common familial environmental factors. One study addressed this issue and estimated that half of TC familiarity was related to genetic factors, the highest of all analysed tumors. After $\mathrm{TC}$, the cancers with the highest genetic component were other endocrine tumours $(28 \%)$, testicular carcinoma (25\%), and breast carcinoma (25\%) [4]. These findings show the important role of genetic alterations in TC development.

One of the most potent risk factors for both sporadic and hereditary medullary thyroid carcinoma (MTC) is a mutation in the RET proto-oncogene. Hereditary MTC, which is a part of multiple endocrine neoplasia 2 (MEN2) syndromes and familial MTC (FMTC), is associated with RET germline mutations. Furthermore, approximately up to $50 \%$ of sporadic MTC possess somatic mutations in RET [5-7]. While genetic factors have a clear basis in MTC development, this type of carcinoma accounts for only 5\% of all TC cases. The majority of TC comprises papillary thyroid carcinoma (PTC) and follicular thyroid carcinoma (FTC), which together are termed as differentiated thyroid carcinoma (DTC) [8]. Data from studies focusing on DTC showed that the Standard Incidence Risk (SIR) of DTC was between 7.8 and 4.1 for male relatives and between 4.9 and 1.9 for female relatives of the proband [9-11]. For PTC, which is the most common histological subtype of TC, the SIR was calculated as 5.8 and 4.1 for male and female relatives, respectively [11]. Although the SIR for DTC was lower overall than for TC, it remains relatively high in comparison to other cancers. For instance, in breast cancer, where well-known BRCA1 
and $B R C A 2$ mutations are prevalent, family risk ratio was assessed at 1.83-2.01 [3].

DTC shows familial occurrence, which means that at least two first-degree relatives of a proband are affected by DTC. Familial DTC accounts for approximately $10 \%$ of all DTC cases. If the genetic background of DTC is strong, linkage analyses should easily uncover predisposing loci in affected families. However, such linkage analyses have identified only a handful of genes; driver mutations existed in single families and they were absent in sporadic DTC [12]. Therefore, it seems that the genetic predisposition to DTC is probably polygenic with low-penetrance genes involved. Such genetic alterations are difficult to uncover by linkage but may be revealed by association studies.

\section{Genome-wide association studies in DTC}

Linkage analysis is the first-choice approach in searching for predisposing genes with traits of a Mendelian pattern of inheritance. However, if low-penetrance genes are involved, this approach may not identify candidate loci and therefore other methods could be employed, such as association studies. Association studies are based on analysis of the allelic distribution between two populations: individuals with a given trait and a control group. By this approach we are able to detect genetic alterations that are more frequent in a tested population and hence are associated with a disease. Association studies are based mainly on analysis of single nucleotide polymorphisms (SNP), but also copy number variants (CNV), repetitive elements, or other more complex genetic alterations. In order to analyse the whole human genome, association studies require genotyping of hundreds of thousands of SNPs. Therefore, association studies are focused on selected fragments of human DNA where putative culprit genes might be located. The selection of such DNA regions is based on the function of genes associated with cancer development pathways, such as cell differentiation and growth, or DNA repair. This strategy, called a "gene candidate approach", relies on a priori knowledge about the location and function of genes involved in the aforementioned pathways. However, in order to uncover novel genes that are important for cancer development, an agnostic screening across the genome must be performed. With the HapMap Project it was estimated that around 500,000 SNPs were required in order to capture most of the genetic variation in the human genome [13]. However, at that time commercially available SNP arrays were largely prohibitive due to their low coverage and high costs. In 1998 a research group from Whitehead Institute from the Massachusetts Institute of Technology published a project using the first SNP microarray containing 500 variants [14]. Five years later a commercial microarray, GeneChip Mapping $10 \mathrm{~K}$ by Affymetrix, already contained 10,000 SNPs; in 2007 microarrays by Affymetrix and Illumina containing 1 million SNPs were available [15]. Together these achievements allowed for the investigation of the entire human genome by association study, ushering in the era of the genome-wide association study (GWAS).

The first GWAS, published in 2005, was a milestone in modern genetics. Genomic DNA from 96 patients with age-related macular degeneration (AMD) versus 50 healthy controls was genotyped on the Affymetrix GeneChip Mapping 100K platform. This uncovered the association of a novel variant, rs380390, located in the intron of the complement factor $\mathrm{H}(\mathrm{CFH})$ gene, with AMD. The odds ratio (OR) carried by rs380390 was 7.4 $\left(\mathrm{p}<10^{-7}\right)$, and it has remained one of the highest for GWAS SNPs [16]. Interestingly, CFH had not been identified as being involved in AMD pathogenesis prior to the rs380390 report. Further research delivered data elucidating the role of CFH in AMD pathogenesis [17-19]. The results of the first GWAS indicated the potential of GWAS in revealing mechanisms of complex diseases.

More than 3600 GWAS studies have been published to date, including eight for DTC. (https://www.ebi. ac.uk/gwas, accessed January 2019). For DTC, 31 associations have been reported (Tab. I) [20-27]. SNPs uncovered by GWAS are located on 12 chromosomes in 17 different loci. Loci with the most "dense" SNPs are $9 q 22$ and $14 q 13$, containing five and four variants, respectively. Interestingly, 9q22 and 14q13 harbour thyroid transcription factors (9q22: FOXE1; 14q13: NKX2-1) that are involved in thyroid development $[21,28]$. The remaining variants are in the vicinity of genes that have not been directly implicated in thyroid function. DTC-associated GWAS SNPs carry a risk varying from 1.20 to 2.09 , with the majority being below 1.50 . Such moderate ORs are consistent with the observations from other cancers, in which the effect of polymorphisms was usually small (OR < 2.0) [29].

GWAS mainly uses platforms containing frequent genetic variants. Therefore, according to the common disease-common variant (CD-CV) theory, the biological effect carried by these polymorphisms should be small [30]. However, regarding the relatively strong genetic component in DTC, their risk seems to be surprisingly moderate. This could be explained in part by a polygenic background, with many low-penetrance genes contributing to DTC development. For example, a DTC polygenic risk model showed that each risk allele of the first five reported GWAS SNPs (rs965513, rs944289, rs966423, rs2439302, and rs116909374) increased the OR for PTC by 1.51 and 1.35 in the United States (US) and Polish populations, respectively [31]. Individuals with 
Table I. Single-nucleotide polymorphisms (SNP) reported by genome-wide association studies (GWAS) conducted in non-medullary thyroid carcinoma (NMTC)

\begin{tabular}{|c|c|c|c|c|c|c|c|}
\hline Region & SNP [risk allele] & Reported gene(s) & $\begin{array}{l}\text { Risk allele } \\
\text { frequency }\end{array}$ & OR & $95 \% \mathrm{CI}$ & $p$ value & Reference \\
\hline $1 q 42.2$ & rs12129938[A] & PCNXL2 & 0.80 & 1.32 & $1.20-1.43$ & 4.00E-11 & [20] \\
\hline $1 q 42.2$ & rs4649295[T] & PCNXL2 & 0.82 & 1.43 & NR & $6.00 \mathrm{E}-08$ & [25] \\
\hline $1 \mathrm{p} 13.3$ & rs4915076[T] & VAV3 & 0.70 & 1.33 & NR & 8.47E-08 & [25] \\
\hline $2 q 35$ & rs966423[C] & DIRC3 & 0.44 & 1.34 & $1.22-1.47$ & $1.30 \mathrm{E}-09$ & [22] \\
\hline $2 q 35$ & rs6759952[T] & DIRC3 & 0.43 & 1.25 & $1.16-1.34$ & $6.40 \mathrm{E}-10$ & [26] \\
\hline $2 q 35$ & rs11693806[C] & DIRC3 & 0.32 & 1.43 & $1.33-1.54$ & 1.50E-24 & [20] \\
\hline $2 q 35$ & rs12990503[G] & DIRC3 & 0.63 & 1.34 & NR & 3.55E-09 & [25] \\
\hline $3 q 26.2$ & rs6793295[T] & LRRC34, TERC & 0.76 & 1.23 & $1.15-1.33$ & 2.70E-08 & [20] \\
\hline $3 p 14.2$ & rs9858271[G] & FHIT & 0.43 & 1.26 & NR & $6.82 \mathrm{E}-07$ & [25] \\
\hline $4 q 21.1$ & rs1874564[G] & SEPT11 & 0.69 & 1.31 & NR & $2.04 \mathrm{E}-07$ & [25] \\
\hline 5p15.33 & rs10069690[T] & TERT & 0.28 & 1.20 & $1.12-1.29$ & $3.20 \mathrm{E}-07$ & [20] \\
\hline $5 q 22.1$ & rs73227498[A] & NREP, EPB41L4A & 0.87 & 1.37 & $1.23-1.49$ & 3.00E-10 & [20] \\
\hline $8 p 12$ & rs2439302[G] & NRG1 & 0.35 & 1.36 & $1.23-1.50$ & $2.00 \mathrm{E}-09$ & [22] \\
\hline 8p12 & rs2466076[G] & NRG1 & 0.48 & 1.32 & $1.23-1.41$ & $1.50 \mathrm{E}-17$ & [20] \\
\hline $8 p 12$ & rs6996585[G] & NRG1 & 0.23 & 1.39 & NR & $1.08 \mathrm{E}-10$ & [25] \\
\hline 9q22.33 & rs965513[A] & FOXE1, PTCSC2 & 0.34 & 1.75 & $1.59-1.94$ & $1.70 \mathrm{E}-27$ & [21] \\
\hline 9q22.33 & rs $965513[A]$ & FOXE1 & 0.33 & 1.65 & $1.43-1.91$ & $4.80 \mathrm{E}-12$ & [23] \\
\hline $9 q 22.33$ & rs10122541[G] & FOXE1 & 0.33 & 1.54 & $1.40-1.70$ & $1.10 \mathrm{E}-17$ & [24] \\
\hline 9q22.33 & rs7037324[A] & FOXE1, C9orf156 & 0.34 & 1.54 & $1.39-1.70$ & $1.20 \mathrm{E}-17$ & [24] \\
\hline 9q22.33 & rs1588635[A] & KRT18P13, FOXE1 & 0.40 & 1.69 & $1.59-1.82$ & $2.00 \mathrm{E}-58$ & [20] \\
\hline 9q22.33 & rs $72753537[\mathrm{C}]$ & FOXE1 & 0.07 & 1.41 & NR & 7.67E-06 & [25] \\
\hline $10 q 24.33$ & rs7902587[T] & OBFC1 & 0.11 & 1.41 & $1.27-1.56$ & $5.40 \mathrm{E}-11$ & [20] \\
\hline $12 q 14.3$ & rs11175834[T] & MSRB3 & 0.15 & 1.37 & NR & $4.26 \mathrm{E}-08$ & [25] \\
\hline $14 q 13.3$ & rs944289[T] & NKX2-1, PTCSC3 & 0.57 & 1.37 & $1.24-1.52$ & $2.00 \mathrm{E}-09$ & [21] \\
\hline $14 q 13.3$ & rs116909374[T] & MBIP, RN7SKP21 & 0.02 & 2.09 & $1.68-2.60$ & 4.60E-11 & [22] \\
\hline $14 q 13.2$ & rs368187[G] & LOC105370452 & 0.58 & 1.39 & $1.30-1.47$ & $5.10 \mathrm{E}-23$ & [20] \\
\hline $14 q 13.2$ & rs34081947[T] & $N K X 2-1$ & 0.41 & 1.27 & NR & 1.19E-07 & [25] \\
\hline $14 q 24.3$ & rs10136427[C] & BATF & 0.87 & 1.40 & $1.23-1.60$ & 4.35E-07 & [27] \\
\hline $15 q 22.33$ & rs2289261[C] & SMAD3 & 0.68 & 1.23 & $1.15-1.32$ & 3.10E-09 & [20] \\
\hline $15 q 22.33$ & rs56062135[T] & SMAD3 & 0.25 & 1.24 & $1.16-1.34$ & 4.90E-09 & [20] \\
\hline $20 q 12$ & rs7267944[C] & DHX35 & 0.23 & 1.39 & $1.24-1.56$ & 2.13E-08 & [27] \\
\hline
\end{tabular}

$\mathrm{OR}$ — odds ratio; $\mathrm{Cl}$ — confidence interval; $\mathrm{NR}$ — non releasable

seven or more risk alleles had ORs calculated at 13.38 and 6.16, respectively. Data from the US and Polish populations were concomitant, although cumulative ORs for the latter were clearly lower. This difference is probably due to population heterogeneity, unknown environmental factors, and/or the fact that Polish controls were younger than cases $[27,31]$.

The additive effect of GWAS markers was also shown in Italian and Han populations. The cumulative effect of 11 independent susceptibility SNPs in an Italian population $(\mathrm{n}=1791$ DTC cases and $\mathrm{n}=1588$ controls $)$ showed an increase of DTC risk by OR $=1.30$ per allele. Individuals with 14 or more risk alleles had a 7.68 times higher risk compared to those with seven or less risk alleles. When rs9655113 was added to the model, the risk of DTC increased to 27.45 [32]. The analysis of the cumulative effect of four GWAS SNPs (rs965513, rs944289, rs966423, and rs2439302) in Chinese PTC cases $(\mathrm{n}=838)$ showed that individuals with more than five risk alleles had an 8.84-fold increased risk of PTC compared to those with one risk allele [33]. Of note, in Ohio, Polish, and Italian populations the 
analysed variants explained only $11 \%, 6 \%$, and $4 \%$ of DTC risk, respectively [31-33]. These data clearly show that there are many undiscovered variants associated with DTC. Furthermore, the cumulative effect of GWAS SNPs suggests that by uncovering novel variants these cumulative risk scores will achieve clinical utility. For example, if we could identify potentially more aggressive forms of DTC using GWAS SNPs genotyped from germline DNA, it would be of great clinical significance in treatment strategies for patients with high risk of a poorer outcome.

\section{Association of GWAS markers with clinical features}

There are several staging systems created to predict clinical outcome in DTC patients. Among them, the Union for International Cancer Control/TNM (UICC/TNM) is the most commonly used because of its relatively stable and replicable prediction of clinical outcome in various populations worldwide [34]. The UICC/TNM describes tumour size (T), and lymph node $(\mathrm{N})$ and distant metastasis $(\mathrm{M})$ presence [35]. Although the UICC/TNM classification is widely accepted, it has some disadvantages. First, it explains (as other DTC staging systems) only a small proportion of thyroid carcinoma-related deaths. Second, this system fails to predict persistence/recurrence of the disease [36]. Thyroid carcinoma is a complex disease with many factors impacting the final outcome such as different histological subtypes, localisation of metastases, molecular profiling, effectiveness of the initial treatment, which are not considered by the TNM system. Despite its limitations, TNM remains the main staging system for DTC. Therefore, studies for clinical associations between GWAS markers and clinical variables have focused primarily on associations with tumour, lymph node, and distant metastases.

\section{T stage}

Tumour diameter measured at the time of diagnosis is the result of many factors, including cancer growth rate, but also others that are not related to cancer biology, such as patient compliance and guidelines for performing a fine-needle aspiration biopsy. Although many factors are involved in tumor growth, one of them is cancer aggressiveness. Two GWAS variants (rs965513 and rs966423) showed association with tumor size at the time of diagnosis (slope of regression 0.14 , and $\mathrm{OR}=1.24$, respectively). These associations were detected in both PTC and DTC populations (Tab. II). Variant rs965513 is located at 9q22.33 in close proximity to Forkhead Box E1 (FOXE1) (also known as Thyroid Transcription factor 2). Follow-up studies showed that rs965513 overlapped with a bidirectional promoter shared by FOXE1 and a large non-coding RNA (lncRNA) gene called Papillary Thyroid Carcinoma Susceptibility Candidate 2 (PTCSC2). The latter was downregulated in PTC tumor tissue, and its suppression was stronger in the presence of the 965513 risk allele [37, 38]. PTCSC2 acted as a tumor suppressor by reverting the promoter inhibition caused by myosin-9 (MYH9) [39]. These findings were fundamental in elucidating the role of rs965513 in PTC predisposition. Rs 965513 is one of the most prominent DTC variants replicated in many populations [40]. Interestingly, it has shown an association with tumour diameter in classical PTC (cPTC, $\mathrm{n}=891)$ but not in follicular variant $\mathrm{PTC}$ (fvPTC, $\mathrm{n}=243$ ) (Tab. II). This may be related to its stronger association with cPTC compared to fvPTC [41]. Such association differences might suggest that PTC is not homogeneous in terms of genetic background, and different genes might be involved in different histological subtypes [42].

\section{N stage}

Spread to local lymph nodes is not a high-risk factor for a poor outcome unless there are multiple large metastases [43-45]. However, the presence of lymph node metastasis always requires radioactive iodine therapy in microPTC [34]. Three SNPs displayed association with N stage (rs965513, rs116909374, and rs2439302). The risk allele of rs 116909374 was associated with nearly $50 \%$ lower relative risk of lymph node metastasis in a large Ohio cohort of 1216 PTC cases. Of note, this association was probably entirely coming from fvPTC cases where rs116909374 was strongly associated with N1, whereas it did not show association with $\mathrm{N}$ stage for cPTC patients. Rs116909374, together with neighbouring rs944289, carried a higher risk for fvPTC compared to CPTC [41]. These data are consistent with a report showing a smaller rate of lymph node metastasis in fvPTC [46]. In a very recent GWAS study analysing the genetic background of thyroid nodules one variant was associated with the trait 9q12: rs4745021, and a second (14q13.3: rs944289) showed a trend towards genome-wide significance [47]. The other GWAS SNPs analysed in the study did not show association with thyroid nodules [47]. SNP rs944289 was associated with benign thyroid tumors in two other populations [48, 49]. This suggests that the genetic backgrounds of benign and malignant thyroid tumors only partially overlap.

\section{M stage}

Distant metastasis is the hallmark of cancer progression and is one of the most important risk factors for DTC-related mortality. So far, there has been only one GWAS variant (rs944289) reported to be associated with M stage. The risk allele of rs944289 was associated with 
Table II. Associations between genome-wide association studies (GWAS) single-nucleotide polymorphisms (SNPs) and clinical features

\begin{tabular}{|c|c|c|c|c|}
\hline SNP & Feature & Risk & $\mathrm{p}$ value & Reference \\
\hline \multicolumn{5}{|l|}{ DTC } \\
\hline rs965513 & $\mathrm{N}$ stage & $\mathrm{OR}=1.59(1.11-2.29)$ & 0.030 & [54] \\
\hline rs944289 & Multifocality & $\mathrm{OR}=0.68(0.53-0.88)$ & 0.003 & [33] \\
\hline \multirow{3}{*}{ rs966423 } & T stage & $\mathrm{OR}=1.24(1.02-2.51)$ & 0.031 & [33] \\
\hline & ETE & $\mathrm{OR}=1.57(1.08-2.30)$ & 0.019 & [33] \\
\hline & Mortality & $\mathrm{HR}=1.60(1.02-2.49)$ & 0.038 & [50] \\
\hline \multicolumn{5}{|l|}{ PTC } \\
\hline \multirow{2}{*}{ rs965513 } & Tumour diameter & Slope of regression $0.14(0.02-0.27)$ & 0.025 & [41] \\
\hline & ETE & $\mathrm{OR}=1.29(1.01-1.67)$ & 0.045 & [41] \\
\hline rs116909374 & N1 stage & $\mathrm{OR}=0.61(0.41-0.90)$ & 0.016 & [41] \\
\hline \multirow{2}{*}{ rs2439302 } & N1 stage & $\mathrm{OR}=1.24(1.04-1.49)$ & 0.016 & [41] \\
\hline & Multifocality & $\mathrm{OR}=1.24(1.05-1.48)$ & 0.012 & [41] \\
\hline \multicolumn{5}{|l|}{ cPTC } \\
\hline rs965513 & Tumour diameter & Slope of regression $0.20(0.06-0.34)$ & 0.006 & [41] \\
\hline rs944289 & M1 stage & $\mathrm{OR}=0.58(0.34-0.98)$ & 0.042 & [41] \\
\hline \multicolumn{5}{|l|}{ fvPTC } \\
\hline rs116909374 & N1 stage & $\mathrm{OR}=0.29(0.07-0.87)$ & 0.049 & [41] \\
\hline rs966423 & Age at time of diagnosis & Slope of regression $2.39(0.02-4.77)$ & 0.048 & [41] \\
\hline rs2439302 & Age at time of diagnosis & Slope of regression $3.36(0.93-5.80)$ & 0.007 & [41] \\
\hline \multicolumn{5}{|l|}{ microPTC } \\
\hline rs2439302 & ETE & $\mathrm{OR}=2.55(1.13-6.33)$ & 0.032 & [41] \\
\hline
\end{tabular}

DTC — differentiated thyroid carcinoma; PTC — papillary thyroid carcinoma; ETE — extrathyroidal extension; OR — odds ratio; HR — hazard ratio

approximately $50 \%$ reduction in the frequency of distant metastases in cPTC (Tab. II). This seems conflicting, but the same SNP might act differently according to different stages of tumor development $[50,51]$. The risk alleles of rs944289 and rs116909374 showed protective action against distant and lymph nodes metastases, respectively.

While the mechanism by which rs116909374 predisposes to DTC remains unknown, more is known about rs944289 and its impact on PTC pathogenesis. This SNP is located $3.2 \mathrm{~kb}$ upstream from a lncRNA called Papillary Thyroid Carcinoma Susceptibility Candidate 3 (PTCSC3). The risk allele weakens the promoter activity caused by the CCAAT/enhancer binding proteins (C/EBP). PTCSC3 was suppressed in PTC tissue, and its suppression was stronger in the presence of rs944289 risk allele [49, 52]. Additionally, rs944289 is located approximately $337 \mathrm{~kb}$ from the NK2 Homeobox 1 (NKX2-1) gene, also known as Thyroid Transcription Factor 1 (TTF1). Although this transcript remains a putative target for the 14q13.3 variants, no such biological link has been proven thus far [21]. Interestingly, the first-reported GWAS loci, 9q22.22 and 14q13.3, contain two lncRNA genes that have been shown to be involved in the development of PTC [37,
52]. Therefore, it seems that dysregulation of lncRNAs might be of great importance in PTC pathogenesis [53].

\section{Extrathyroidal extension and multifocality}

Two variants (rs965513 and rs966423) have shown an association with ETE (Tab. II). These variants seem to be the most interesting in terms of clinical associations. Rs965513 was also associated with tumor diameter, N1 stage, and lack of lymphocytic infiltration [41, 54]. The latter has been associated with a favourable prognosis with a diminished recurrence rate [55]. Rs966423 showed association with the overall mortality, which is discussed in greater detail below [50].

SNPs rs944289 and rs24390302 were associated with multifocality [33, 41]. Multifocality may increase the risk of lymph node metastases, which in turn is important for determining treatment [56]. Rs944289 has been linked with multifocality in Asian PTC patients $(n=838)$ [33]. In PTC cases from central Ohio $(n=1216)$ no association with multifocality for rs 944289 was detected [41]; this might reflect a different impact of GWAS on the clinical course depending on the population, but such findings need to be further validated. 


\section{Mortality}

Overall cancer-related mortality is the ultimate measure of clinical outcome in malignant diseases. In a large study of DTC cases $(n=1826)$, five GWAS variants were analysed (rs116909374, rs965513, rs944289, rs966423, and rs2439302) for their association with clinical features, including overall mortality [50]. The median follow-up in the study was 8.7 years with the overall mortality of 85 cases $(4.63 \%)$, including 53 deaths for CPTC $(3.71 \%)$, 15 for fvPTC (6.17\%), 10 for FTC (35\%), and seven for insular FTC (35\%). Only one variant displayed association with poor clinical outcome. Genotype "TT" vs. genotypes "CT" and "CC" of rs966423 was associated with overall increased mortality (Tab. II). The mortality rate was twice as high in patients with the risk genotype (6.4\% vs. 3.7\%). Rs966423 showed association with mortality regardless of the other risk factors such as ETE, distant metastases, and angiovascular invasion. Rs966423 is located at 2q35 locus within a lncRNA called Disrupted In Renal Carcinoma 3 (DIRC3). DIRC3 was originally identified in 2003 as a fusion transcript involved in familial renal carcinoma [57]; however, there have been no data showing involvement of DIRC3 in DTC pathogenesis. Similar to the association of rs944289 with distant metastases, it was not the risk allele of rs966423 that displayed association with mortality. These findings underscore the complexity of DTC genetics with variants acting differentially depending on stage of DTC development, but also suggests the presence of additional undiscovered disease-associated variants.

\section{Conclusions}

Genetic predisposition to DTC seems to be relatively strong based on population case-control studies. So far, eight GWASs conducted in DTC have yielded more than 31 genetic DTC-associated variants. It has been shown that the cumulative genetic risk score increases by adding variants. Hence, it is likely that multiple low penetrance genes are involved in DTC. Hopefully, the discovery of more DTC-associated SNPs will improve cumulative risk modelling, resulting in significant utility for clinicians and patients alike.

Only a few studies have analysed association between GWAS markers and clinical features. Such studies are difficult due to differences in clinical guidelines, heterogeneity of populations, the proportions of DTC histological subtypes, and more. Despite a low risk of variants for DTC predisposition, several interesting clinical associations have been detected in different populations worldwide. Markers associated with DTC have shown association with tumor diameter (rs965513, rs966423), N stage (rs965513, rs116909374, rs2439302),
M stage (rs944289), ETE (rs965513, rs966423), and multifocality (rs944289, rs2439302). One variant (rs966423) showed association with increased mortality in DTC cases, whereas the DTC risk allele for rs966423 was associated with favourable outcome. Overall, these studies demonstrate that genetic markers not only carry a risk for DTC, but they also might influence the clinical course of the disease.

\section{Funding}

National Cancer Institute Grants P30CA16058 and P50CA168505 to A.d.l.C. and the American Thyroid Association Thy-Ca Research Grant to J.J.

\section{References}

1. Goldgar DE, Easton DF, Cannon-Albright LA, et al. Systematic population-based assessment of cancer risk in first-degree relatives of cancer probands. J Natl Cancer Inst. 1994; 86(21): 1600-1608, doi: 10.1093/jnci/86.21.1600, indexed in Pubmed: 7932824 .

2. Dong C, Hemminki K. Modification of cancer risks in offspring by sibling and parental cancers from 2,112,616 nuclear families. Int J Cancer. 2001; 92(1): 144-150, indexed in Pubmed: 11279618.

3. Risch N. The genetic epidemiology of cancer: interpreting family and twin studies and their implications for molecular genetic approaches. Cancer Epidemiol Biomarkers Prev. 2001; 10(7): 733-741, indexed in Pubmed: 11440958.

4. Czene K, Lichtenstein P, Hemminki K. Environmental and heritable causes of cancer among 9.6 million individuals in the Swedish Family-Cancer Database. Int J Cancer. 2002; 99(2): 260-266, doi: 10.1002/ijc.10332, indexed in Pubmed: 11979442.

5. Eng C, Clayton D, Schuffenecker I, et al. The relationship between specific RET proto-oncogene mutations and disease phenotype in multiple endocrine neoplasia type 2. International RET mutation consortium analysis. JAMA. 1996; 276(19): 1575-1579, indexed in Pubmed: 8918855.

6. Donis-Keller H, Dou S, Chi D, et al. Mutations in the RET proto-oncogene are associated with MEN 2A and FMTC. Hum Mol Genet. 1993; 2(7): 851-856, doi: 10.1093/hmg/2.7.851, indexed in Pubmed: 8103403.

7. Marsh D, Learoyd D, Andrew S, et al. Somatic mutations in theRETproto-oncogene in sporadic medullary thyroid carcinoma. Clini Endocrinol (Oxf). 1996; 44(3): 249-257, doi: 10.1046/j.1365-2265.1996.681503.x, indexed in Pubmed: 8729519.

8. Jarząb B, Dedecjus M, Handkiewicz-Junak D, et al. Diagnostics and Treatment of Thyroid Carcinoma. Endokrynol Pol. 2016; 67(1): 74-107, doi: 10.5603/EP.2016.0011, indexed in Pubmed: 26884119.

9. Hemminki K, Dong C. Familial relationships in thyroid cancer by histo-pathological type. Int J Cancer. 2000; 85(2): 201-205, indexed in Pubmed: 10629078.

10. Hrafnkelsson J, Tulinius H, Jónasson JG, et al. Familial non-medullary thyroid cancer in Iceland. J Med Genet. 2001; 38(3): 189-191, doi: 10.1136/jmg.38.3.189, indexed in Pubmed: 11303513.

11. Frich L, Glattre E, Akslen, A. Familial occurrence of nonmedullary thyroid cancer: a population-based study of 5673 first-degree relatives of thyroid cancer patients from Norway. Cancer Epidemiol Biomarkers Prev. 2001; 10(2): 113-117, indexed in Pubmed: 11219767.

12. Peiling Yang S, Ngeow J. Familial non-medullary thyroid cancer: unraveling the genetic maze. Endocr Relat Cancer. 2016; 23(12): R577-R595, doi: 10.1530/ERC-16-0067, indexed in Pubmed: 27807061.

13. International HapMap Consortium. A haplotype map of the human genome. Nature. 2005; 437(7063): 1299-1320, doi: 10.1038/nature04226, indexed in Pubmed: 16255080.

14. Wang DG. Large-Scale Identification, Mapping, and Genotyping of Single-Nucleotide Polymorphisms in the Human Genome. Science. 1998; 280(5366): 1077-1082, doi: 10.1126/science.280.5366.1077, indexed in Pubmed: 9582121.

15. LaFramboise T. Single nucleotide polymorphism arrays: a decade of biological, computational and technological advances. Nucleic Acids Res. 2009; 37(13): 4181-4193, doi: 10.1093/nar/gkp552, indexed in Pubmed: 19570852 .

16. Klein RJ, Zeiss C, Chew EY, et al. Complement factor $\mathrm{H}$ polymorphism in age-related macular degeneration. Science. 2005; 308(5720): 385-389, doi: 10.1126/science.1109557, indexed in Pubmed: 15761122.

17. Thakkinstian A, Han P, McEvoy M, et al. Systematic review and meta-analysis of the association between complement factor $\mathrm{H} \mathrm{Y} 402 \mathrm{H}$ polymorphisms and age-related macular degeneration. Hum Mol 
Genet. 2006; 15(18): 2784-2790, doi: 10.1093/hmg/ddl220, indexed in Pubmed: 16905558.

18. Despriet DDG, Klaver CCW, Witteman JCM, et al. Complement factor H polymorphism, complement activators, and risk of age-related macular degeneration. JAMA. 2006; 296(3): 301-309, doi: 10.1001/jama.296.3.301, indexed in Pubmed: 16849663.

19. Shaw PX, Zhang Li, Zhang M, et al. Complement factor $\mathrm{H}$ genotypes impact risk of age-related macular degeneration by interaction with oxidized phospholipids. Proc Natl Acad Sci USA. 2012; 109(34): 13757-13762, doi: 10.1073/pnas.1121309109, indexed in Pubmed: 22875704.

20. Gudmundsson J, Thorleifsson G, Sigurdsson JK, et al. A genome-wide association study yields five novel thyroid cancer risk loci. Nat Commun. 2017; 8: 14517, doi: 10.1038/ncomms14517, indexed in Pubmed: 28195142

21. Gudmundsson J, Sulem P, Gudbjartsson DF, et al. Common variants on 9q22.33 and 14q13.3 predispose to thyroid cancer in European populations. Nat Genet. 2009; 41(4): 460-464, doi: 10.1038/ng.339, indexed in Pubmed: 19198613.

22. Gudmundsson J, Sulem P, Gudbjartsson DF, et al. Discovery of common variants associated with low TSH levels and thyroid cancer risk. Nat Genet. 2012; 44(3): 319-322, doi: 10.1038/ng.1046, indexed in Pubmed: 22267200.

23. Takahashi M, Saenko VA, Rogounovitch TI, et al. The FOXE1 locus is a major genetic determinant for radiation-related thyroid carcinoma in Chernobyl. Hum Mol Genet. 2010; 19(12): 2516-2523, doi: 10.1093/hmg/ddq123, indexed in Pubmed: 20350937.

24. Mancikova V, Cruz R, Inglada-Pérez L, et al. Thyroid cancer GWAS identifies 10q26.12 and 6q14.1 as novel susceptibility loci and reveals genetic heterogeneity among populations. Int J Cancer. 2015; 137(8): 1870-1878, doi: 10.1002/ijc.29557, indexed in Pubmed: 25855579.

25. Son HY, Hwangbo Y, Yoo SK, et al. Genome-wide association and expression quantitative trait loci studies identify multiple susceptibility loci for thyroid cancer. Nat Commun. 2017; 8: 15966, doi: 10.1038/ncomms15966, indexed in Pubmed: 28703219.

26. Köhler A, Chen B, Gemignani F, et al. Genome-wide association study on differentiated thyroid cancer. J Clin Endocrinol Metab. 2013; 98(10): E1674-E1681, doi: 10.1210/jc.2013-1941, indexed in Pubmed: 23894154.

27. Figlioli G, Köhler A, Chen B, et al. Novel genome-wide association study-based candidate loci for differentiated thyroid cancer risk. J Clin Endocrinol Metab. 2014; 99(10): E2084-E2092, doi: 10.1210/jc.2014-1734, indexed in Pubmed: 25029422.

28. Kimura S. Thyroid-specific transcription factors and their roles in thyroid cancer. J Thyroid Res. 2011; 2011: 710213, doi: 10.4061/2011/710213, indexed in Pubmed: 21687604.

29. Visscher PM, Wray NR, Zhang Q, et al. 10 Years of GWAS Discovery: Biology, Function, and Translation. Am J Hum Genet. 2017; 101(1): 5-22, doi: 10.1016/j.ajhg.2017.06.005, indexed in Pubmed: 28686856.

30. Iyengar SK, Elston RC. The genetic basis of complex traits: rare variant or "common gene, common disease"? Methods Mol Biol. 2007; 376: 71-84, doi: 10.1007/978-1-59745-389-9 6, indexed in Pubmed: 17984539

31. Liyanarachchi S, Wojcicka A, Li W, et al. Cumulative risk impact of five genetic variants associated with papillary thyroid carcinoma. Thyroid. 2013; 23(12): 1532-1540, doi: 10.1089/thy.2013.0102, indexed in Pubmed: 23659773

32. Figlioli G, Chen B, Elisei R, et al. Novel genetic variants in differentiated thyroid cancer and assessment of the cumulative risk. Sci Rep. 2015; 5 8922, doi: 10.1038/srep08922, indexed in Pubmed: 25753578.

33. Wei WJ, Lu ZW, Wang Yu, et al. Clinical significance of papillary thyroid cancer risk loci identified by genome-wide association studies. Cancer Genet. 2015; 208(3): 68-75, doi: 10.1016/j.cancergen.2015.01.004, indexed in Pubmed: 25746573.

34. Haugen BR. 2015 American Thyroid Association Management Guidelines for Adult Patients with Thyroid Nodules and Differentiated Thyroid Cancer: What is new and what has changed? Cancer. 2017; 123(3): 372-381, doi: 10.1002/cncr.30360, indexed in Pubmed: 27741354

35. Mankarios D, Baade P, Youl P, et al. Validation of the QTNM staging system for cancer-specific survival in patients with differentiated thyroid cancer. Endocrine. 2014; 46(2): 300-308, doi: 10.1007/s12020-013-0078-9, indexed in Pubmed: 24174176.

36. Yang L, Shen W, Sakamoto N. Population-based study evaluating and predicting the probability of death resulting from thyroid cancer and other causes among patients with thyroid cancer. J Clin Oncol. 2013; 31(4) 468-474, doi: 10.1200/JCO.2012.42.4457, indexed in Pubmed: 23270002

37. He H, Li W, Liyanarachchi $\mathrm{S}$, et al. Genetic predisposition to papillary thyroid carcinoma: involvement of FOXE1, TSHR, and a novel lincRNA gene, PTCSC2. J Clin Endocrinol Metab. 2015; 100(1): E164-E172, doi: 10.1210/jc.2014-2147, indexed in Pubmed: 25303483.

38. He H, Li W, Liyanarachchi S, et al. Multiple functional variants in long-range enhancer elements contribute to the risk of SNP rs965513 in thyroid cancer. Proc Natl Acad Sci USA. 2015; 112(19): 6128-6133, doi: 10.1073/pnas.1506255112, indexed in Pubmed: 25918370.

39. Wang Y, He H, Li W, et al. MYH9 binds to lncRNA gene PTCSC2 and regulates FOXE1 in the 9q22 thyroid cancer risk locus. Proc Natl Acad
Sci USA. 2017; 114(3): 474-479, doi: 10.1073/pnas.1619917114, indexed in Pubmed: 28049826

40. Saenko VA, Rogounovitch TI. Genetic Polymorphism Predisposing to Differentiated Thyroid Cancer: A Review of Major Findings of the Genome-Wide Association Studies. Endocrinol Metab (Seoul). 2018; 33(2): 164-174, doi: 10.3803/EnM.2018.33.2.164, indexed in Pubmed: 29947173

41. Jendrzejewski J, Liyanarachchi S, Nagy R, et al. Papillary Thyroid Carcinoma: Association Between Germline DNA Variant Markers and Clinical Parameters. Thyroid. 2016; 26(9): 1276-1284, doi: 10.1089/thy.2015.0665, indexed in Pubmed: 27342578.

42. Jendrzejewski J, Liyanarachchi S, Eiterman A, et al. Fine mapping of $14 \mathrm{q} 13$ reveals novel variants associated with different histological subtypes of papillary thyroid carcinoma. Int J Cancer. 2019; 144(3): 503-512, doi: 10.1002/ijc.31933, indexed in Pubmed: 30350351.

43. Randolph GW, Duh QY, Heller KS, et al. American Thyroid Association Surgical Affairs Committee's Taskforce on Thyroid Cancer Nodal Surgery. The prognostic significance of nodal metastases from papillary thyroid carcinoma can be stratified based on the size and number of metastatic lymph nodes, as well as the presence of extranodal extension. Thyroid. 2012; 22(11): 1144-1152, doi: 10.1089/thy.2012.0043, indexed in Pubmed: 23083442

44. Podnos YD, Smith D, Wagman LD, et al. The implication of lymph node metastasis on survival in patients with well-differentiated thyroid cancer Am Surg. 2005; 71(9): 731-734, indexed in Pubmed: 16468507.

45. Adam MA, Pura J, Goffredo P, et al. Presence and Number of Lymph Node Metastases Are Associated With Compromised Survival for Patients Younger Than Age 45 Years With Papillary Thyroid Cancer. J Clin Oncol. 2015; 33(21): 2370-2375, doi: 10.1200/JCO.2014.59.8391, indexed in Pubmed: 26077238.

46. Yip, L, Nikiforova MN, Yoo JY, et al. Tumor genotype determines phenotype and disease-related outcomes in thyroid cancer: a study of 1510 patients. Ann Surg. 2015 ; 262(3 ): 519-525; discussion 524-525, doi: 10.1097/SLA.0000000000001420, indexed in Pubmed: 26258321

47. Hwangbo Y, Lee E, Son HY, et al. Genome-Wide Association Study Reveals Distinct Genetic Susceptibility of Thyroid Nodules From Thyroid Cancer. J Clin Endocrinol Metab. 2018; 103(12): 4384-4394, doi: 10.1210/jc.2017-02439, indexed in Pubmed: 30099483.

48. Wang YL, Feng SH, Guo SC, et al. Confirmation of papillary thyroid cancer susceptibility loci identified by genome-wide association studies of chromosomes 14q13, 9q22, 2q35 and 8p12 in a Chinese population. Med Genet. 2013; 50(10): 689-695, doi: 10.1136/jmedgenet-2013-101687, indexed in Pubmed: 23847140

49. Rogounovitch TI, Bychkov A, Takahashi M, et al. The common genetic variant rs944289 on chromosome 14q13.3 associates with risk of both malignant and benign thyroid tumors in the Japanese population. Thyroid. 2015; 25(3): 333-340, doi: 10.1089/thy.2014.0431, indexed in Pubmed: 25562676

50. Świerniak M, Wójcicka A, Czetwertyńska M, et al. Association between GWAS-Derived rs966423 Genetic Variant and Overall Mortality in Patients with Differentiated Thyroid Cancer. Clin Cancer Res. 2016, 22(5): 1111-1119, doi: 10.1158/1078-0432.CCR-15-1746, indexed in Pubmed: 26490305

51. Zhao L, Zhang Q, Luan X, et al. STAT3 and STAT5b polymorphism contributes to breast cancer risk and clinical outcomes. Int J Clin Exp Pathol. 2015; 8(2): 2033-2038, indexed in Pubmed: 25973100.

52. Jendrzejewski J, He H, Radomska HS, et al. The polymorphism rs944289 predisposes to papillary thyroid carcinoma through a large intergenic noncoding RNA gene of tumor suppressor type. Proc Natl Acad Sci USA. 2012; 109(22): 8646-8651, doi: 10.1073/pnas.1205654109, indexed in Pubmed: 22586128

53. Liyanarachchi S, Li W, Yan P, et al. Genome-Wide Expression Screening Discloses Long Noncoding RNAs Involved in Thyroid Carcinogenesis. J Clin Endocrinol Metab. 2016; 101(11): 4005-4013, doi: 10.1210/jc.2016-1991, indexed in Pubmed: 27459529.

54. Penna-Martinez M, Epp F, Kahles H, et al. FOXE1 association with differentiated thyroid cancer and its progression. Thyroid. 2014; 24(5) 845-851, doi: 10.1089/thy.2013.0274, indexed in Pubmed: 24325646.

55. Cunha LL, Morari EC, Guihen AC, et al. Infiltration of a mixture of different immune cells may be related to molecular profile of differentiated thyroid cancer. Endocr Relat Cancer. 2012; 19(3): L31-L36, doi: 10.1530/ERC-11-0285, indexed in Pubmed: 22461634.

56. Al Afif A, Williams BA, Rigby MH, et al. Multifocal Papillary Thyroid Cancer Increases the Risk of Central Lymph Node Metastasis. Thyroid. 2015; 25(9): 1008-1012, doi: 10.1089/thy.2015.0130, indexed in Pubmed: 26161997.

57. Bodmer D, Schepens M, Eleveld MJ, et al. Disruption of a novel gene, DIRC3, and expression of DIRC3-HSPBAP1 fusion transcripts in a case of familial renal cell cancer and $\mathrm{t}(2 ; 3)(\mathrm{q} 35 ; \mathrm{q} 21)$. Genes Chromosomes Cancer. 2003; 38(2): 107-116, doi: 10.1002/gcc.10243, indexed in Pubmed: 12939738. 